\title{
Actualización: Trastornos de la alimentación (primera entrega)
}

\author{
Eating disorders (first part)
}

Esteban Kuten*

\begin{abstract}
Resumen
Los trastornos alimentarios son sindromes multicausales que afectan especialmente a mujeres adolescentes. Las manifestaciones clínicas abarcan la conducta, el humor, el discurso y las relaciones familiares, así como la antropometría y las alteraciones metabólicas y endócrinas. Son frecuentes las complicaciones y las comorbilidades. Este primer artículo desarrolla la epidemiología, la sistemática de estudio, la presentación clínica, el diagnóstico y el pronóstico de los trastornos alimentarios.

\section{Abstract}

Eating disorders are multicausal syndromes that specially affect adolescent women. Clinical manifestations include conduct, humor, speech, family relations, antropometric deviations and metabolic alterations. Complications and co-morbidities are frequent. This first article describes etiology, epidemiology, clinical presentation, diagnostic approach and prognosis of eating disorders.
\end{abstract}

Palabras claves: trastornos alimentarios, bulimia nerviosa, anorexia nerviosa, trastornos alimentarios no específicos, atracón. Key words: eating disorders, bulimia nervosa, anorexia nervosa, non specific eating disorders, binge.

Kuten E. Trastornos de la alimentación (primera entrega). Evid. actual. práct. ambul; 10(2): 51-54, mar-abr.2007.

\section{Introducción}

Los trastornos alimentarios TA son entidades muy frecuentes ${ }^{1}$. Suelen comenzar en la adolescencia y predominar en el sexo femenino, aunque cada vez son más frecuentes también en otras subpoblaciones'.

Suelen observarse muchos cuadros "grises" o que se acercan a la conducta alimentaria "normal" lo que dificulta su reconocimiento. De este, modo se corre el riesgo de sobre diagnosticar formas menores y de demorar los tratamientos. Son enfermedades multicausales y en la gran mayoría de los casos se encuentran trastornos comórbidos. Si bien son entidades tratables, su mortalidad y riesgo de recaída son altos.

\section{Definición}

Dentro de esta entidad, se ubican la anorexia nerviosa (AN) la bulimia nerviosa (BN) y los trastornos alimentarios no específicos (TANE).

Anorexia significa en griego "pérdida de apetito". Es un síndrome caracterizado por un rechazo a mantener el peso corporal mínimamente normal, un ayuno deliberado y autoimpuesto; persecución implacable de la delgadez o temor a la gordura y signos y síntomas producto de la inanición ${ }^{2}$. Con frecuencia se asocia a distorsión de la imagen corporal. Se describen dos subtipos: una forma restrictiva presente en casi el $50 \%$ de los casos en que la ingesta está francamente disminuida y acompañada de hiperactividad física pero no purgas ni atracones, y una segunda forma compulsiva-purgativa en la que las purgas o atracones representan la "compensación" para eliminar las calorías ganadas. En general, se trata de vómitos autoprovocados, abuso de laxantes, enemas y, con menor frecuencia, diuréticos y eméticos.

Bulimia significa en griego "hambre de buey". Comparte con la anorexia, la preocupación patológica por el peso y la silueta corporal. Sin embargo, la bulimia se caracteriza por la presencia de episodios de hiperorexia incontrolable y conductas compensatorias a través de vómitos y laxantes ( $80 \%$ de los casos) $\mathrm{u}$ otras formas como el ejercicio intenso $(20 \%)^{1}$. Esta hiperorexia, denominada en gran parte de la literatura como atracón o "binge" presenta desafíos en su definición, la que fue evolucionando con el tiempo. Ver cuadro 1.
Cuadro 1: definición de atracón.

- Crisis consistente en la ingestión masiva y casi frenética de una gran cantidad de alimentos.

- La duración es discreta (por ejemplo, dos horas) y la una cantidad de alimentos es muy superior a la que consumiría la mayoría de las personas en un lapso similar y en circunstancias semejantes.

- El criterio temporal del proceso se incluye para excluir el "picoteo".

- En general, las crisis son a escondidas y fuera del desayuno, almuerzo, merienda o cena. Suelen interrumpirse cuando llega alguien.

- Es brusco, imperativo e ininterrumpido hasta el malestar físico o el vómito.

- El paciente siente que no puede impedirlos o detenerlos.

- Siempre predomina la cantidad sobre la calidad y la necesidad de engullir sobre el placer del gusto.

\section{Criterios diagnósticos}

\section{Anorexia nerviosa}

El ManualDiagnóstico y Estadístico de los Trastornos Mentales $^{3}$ (DSMIVTR) establece los siguientes criterios diagnósticos: a) rechazo a mantener el peso corporal a nivel o por encima del mínimo normal según edad y talla $(85 \%$ del correspondiente o índice de masa corporal menor a 18); b) miedo intenso a ganar peso o volverse obeso, incluso estando por debajo del peso normal; c) alteración de la percepción del peso o la silueta corporal, exageración de su importancia en la autoevalua-ción o negación del peligro que comporta el bajo peso; d) en mujeres pospuberales, ausencia de tres ciclos menstruales consecutivos con respuesta positiva a la administración de hormonas.

Se definen dos subtipos según el tipo de conducta compensatoria inapropiada $(\mathrm{CCl})$ predominante (ver cuadro 2$)$ : en el restrictivo la pérdida de peso se debe básicamente a $\mathrm{CCl}$ restrictivas con ausencia atracones o $\mathrm{CCl}$ purgativas; y en el compulsivo/purgativo hay recurrencia regular de atracones o $\mathrm{CCl}$ purgativas.

\footnotetext{
* Médico Especialista en Medicina Familiar y en Psiquiatría. Servicio de Medicina Familiar y Comunitaria del Hospital Italiano de Buenos Aires.
} 
Cuadro 2: tipos de conductas compensatorias inapropiadas. - Restrictivas: dieta o ejercicio.

- Purgativas: provocación del vómito, uso inapropiado excesivo de laxantes, diuréticos o enemas.

\section{Bulimia nerviosa}

EI DSM IV TR la define como: a) la presencia de atracones recurrentes; b) $\mathrm{CCl}$ reiterada destinada a evitar el aumento de peso; c) repetición de a y b dos veces por semana durante tres meses; d) autoevaluación supeditada en forma indebida al peso y la silueta; e) la alteración no ocurre sólo durante episodios de anorexia nerviosa.

Define dos subtipos según las $\mathrm{CCl}$ : purgativo y no purgativo.

\section{Trastornos alimentarios no específicos}

Esta categoría incluye trastornos de la conducta alimentaria que no cumplen con los criterios para ningún trastorno específico, como por ejemplo: los criterios de anorexia nerviosa pero sin amenorrea o con el peso dentro de los límites de la normalidad; o los de bulimia nerviosa pero con menor frecuencia o duración de los atracones y las $\mathrm{CCl}$; uso regular de $\mathrm{CCl}$ en personas con peso normal luego de ingerir porciones reducidas de alimento (vómitos inducidos después de comer dos galletas); masticar y expulsar sin tragar cantidades considerables de alimentos; trastorno por atracón (atracones recurrentes en ausencia de $\mathrm{CCl}$ ).

\section{Etiología y patogenia}

Una serie de factores predisponentes determinarían cierta vulnerabilidad, algunos eventos precipitantes desencadenarían el cuadro clínico y después actuarían afianzando la entidad los factores de mantenimiento ${ }^{4}$. Ver figura 1.

Los factores genéticos contribuirían con múltiples efectos sobre el temperamento, la personalidad, las formas de regular el humor y la predisposición al ejercicio físico. En presencia de un familiar con un trastorno alimentario, el riesgo de desarrollarlo es 20 veces mayor que el de la población general ${ }^{5}$ y existe 50 a $80 \%$ de concordancia en gemelos monocigotas. Se ha documentado mayor frecuencia de antecedentes familiares de depresión, adicción a alcohol o drogas. Los trastornos del humor, de ansiedad y particularmente el trastorno obsesivo compulsivo a temprana edad, parecerían vincularse a una mayor vulnerabilidad para el desarrollo posterior de un trastorno alimentario, especialmente de anorexia nerviosa ${ }^{5}$.

Ciertos estilos de funcionamiento familiar facilitarían el desarrollo de un trastorno alimentario y/o dificultarían la recuperación de un paciente. A las familias de bulímicos, muchos autores las describen como desorganizadas; y a las de los anoréxicos, sobreprotectoras, con problemas de ansiedad y ejerciendo control en la búsqueda de una "persona ideal", sin promover ni facilitar las experiencias de individuación y separación propias de la adolescencia ${ }^{6}$.

Además, la sociedad y los medios de comunicación de masas tendrían un papel activo a través de su sobrevaloración de la delgadez y de la estigmatización de la obesidad.

Entre los factores de mantenimiento se describen el refuerzo de la valoración social de la delgadez. El éxito obtenido con la disminución del peso sería la primera sensación de un efectivo autocontrol en el contexto de una pubertad que abruma. Los atracones pueden surgir como respuesta al importante apetito, a la frustración y, en estados de humor disfórico, como intentos por sofocar o calmar diferentes estados emocionales. Los episodios de purga pueden acontecer en períodos disociativos en que el paciente busca alejarse de pensamientos negativos. La bulimia nerviosa buscaría "gestionar" estados disfóricos mediante sucesivos atracones y purgas.

Figura 1: modelo Biopsicosocial para la anorexia nerviosa modificado de Lucas A. Anorexia nervosa: Historical background and biopsychosocial determinants. Semin Adolesc Med 1986; 1:1-9 y Lenoir M Anorexia nerviosa en niños y adolescentes Parte1. Arch Argent Pediatr 2006; 104:253-60.

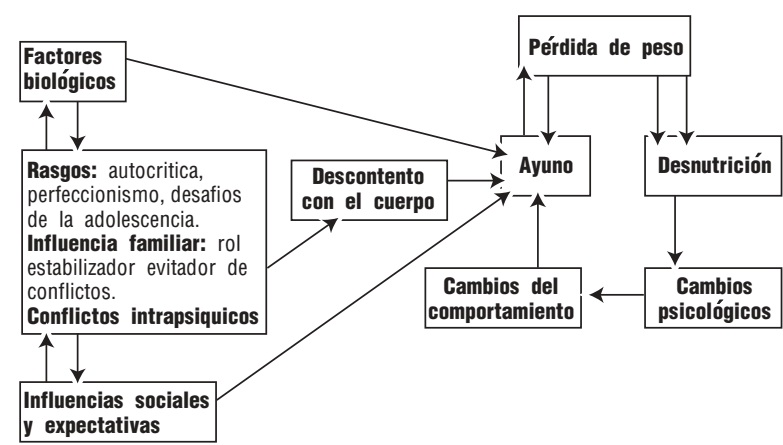

\section{Epidemiología}

Habría un $15 \%$ de individuos con comportamiento alimentario saludable y sin preocupación por el peso o la silueta corporal un $70 \%$ de personas preocupadas por su peso/imagen y otro $15 \%$ con un trastorno alimentario que compromete su normal desarrollo o vida diaria.

Solo 5 a $15 \%$ de los pacientes con anorexia nerviosa y bulimia son varones, alcanzando el $40 \%$ de los que padecen TANE ${ }^{1}$ Habría un aumento de la incidencia en las últimas décadas, tanto en adolescentes y adultos jóvenes, como en edades cada vez más tempranas y avanzadas. La prevalencia es mayor en sociedades industrializadas y abarca a todos los grupos socioeconómicos $^{8}$. Se la estima en 1 a $2 \%$ para anorexia nerviosa y en 2 a $18 \%$ para bulimia. En poblaciones de riesgo como bailarinas, atletas y modelos, la de anorexia llega a 7 u $8 \%$. Los TA son la primera causa de muerte no traumática en menores de 20 años y la tercera causa de enfermedad crónica en adolescentes, por detrás del asma y la obesidad ${ }^{9}$. En los hombres homosexuales habría una mayor prevalencia vinculada a ciertas normas de delgadez de algunas comunidades y, por el contrario, ciertas comunidades lesbianas serían más tolerantes respecto que las mujeres heterosexuales respecto a peso o la forma del cuerpo.

Los pacientes con TANE representan 30 a $50 \%$ de las admisiones a los programas de trastornos de la conducta alimentaria. Los trastornos por atracón estarían presentes en el $25 \%$ de pacientes que solicita una consulta médica por obesidad y en el 50 al $75 \%$ de los obesos con índice de masa corporal superior a 35 .

Los TA suelen estar asociados a dos a cuatro diagnósticos comórbidos.

\section{Presentación clínica}

El motivo de consulta puede estar vinculado con complicaciones físicas o con síntomas iniciales inespecíficos (astenia vértigos o pérdida de energía $\left.{ }^{10}\right)$. Por eso es necesario que en 
las consultas eventuales dentro de distintas áreas de los servicios de salud se indague a los adolescentes sobre los trastornos alimentarios, ya que, teniendo en cuenta el estrecho vínculo entre delgadez y belleza, el diagnóstico no es sencillo. El $70 \%$ de las mujeres jóvenes no sólo desea ser delgada sino que también ha practicado alguna dieta, por lo que habrá que diferenciar a quienes tienen preocupación por su aspecto físico y/o por su peso, de aquellos que tienen un trastorno de la conducta alimentaria. La dificultad diagnóstica se incrementa por la resistencia y la ambivalencia de estos pacientes para relatar sus síntomas, pues temen ser forzados a ganar peso, ser despreciados o sentirse avergonzados.

\section{Anorexia nerviosa}

Si bien se describe una tríada sintomática de adelgazamiento, anorexia y amenorrea, ésta es más evidente en los casos más avanzados. Además, suelen ser pacientes que minimizan o niegan los síntomas ${ }^{3}$.

En una primera fase se describe el inicio del cuadro en forma reactiva a algún acontecimiento (primera relación sexual, cambio de residencia, escolaridad, duelo, decepción, etc.). La conducta restrictiva se instala con el pretexto de adelgazar, aumentando luego con las alteraciones del humor, generalmente depresivas. En una segunda fase, mayormente optimista, aparece un discurso "lógico" que calma la inquietud de los padres, quienes se vuelven poco a poco "cómplices" para evitar la aparición de conflictos. La restricción alimentaria y la delgadez refuerzan el sentimiento de control de sí mismo y de los demás. La tercera fase puede manifestarse a través de una hiperactividad que no logra esconder el ánimo depresivo y la angustia, con períodos de mejorías mínimas. Puede conducir a la anorexia crónica, a trastornos "orgánicos" y a cuadros de caquexia extrema.

El adelgazamiento puede ser muy importante, inclusive llegar a veces hasta más del $50 \%$ del peso normal para la edad. El cuerpo puede ser exhibido o, por el contrario, escondido bajo ropas amplias. De modo constante, los pacientes ignoran su delgadez reflejando el trastorno de alteración de la propia imagen corporal, negando la gravedad de la situación y el peligro al que se exponen. Los pocos que reconocen su delgadez tendrían un pronóstico más favorable.

No son raros los edemas (con albuminemia normal) el cabello seco y opaco; y las uñas estriadas y frágiles. Algunos pacientes adquieren un color amarillento en la piel que se asociaría a hipercarotenemia y es frecuente la hipertricosis con aparición de lanugo. Ante el vómito autoprovocado pueden observarse callosidades en las manos por traumatismos reiterados con la arcada dentaria o "signo de Russell".

Se ven y dibujan más gruesos de lo que son, se pesan luego de las comidas y verifican el valor calórico de los alimentos. Pueden tener fijaciones de tipo dismorfofóbicas respecto de ciertas partes del cuerpo o la cara ${ }^{7}$, acompañándose esta distorsión de sentimientos de aversión al cuerpo y anhedonia.

La potomanía (impulso obsesivo de ingerir líquidos) puede conducir a ingerir decenas de litros por día, ocasionando hiponatremia dilucional. Las purgas pueden determinar trastornos renales o intestinales con riesgo de vida por los trastornos electrolíticos: hiponatremia, hipomagnesemia, hipocalcemia e hipofosfatemia.

Las manifestaciones del aparato digestivo incluyen alteraciones en la secreción salival, erosión del esmalte dental, gingivitis y pérdida de piezas dentarias, así como sensación de plenitud gástrica y constipación grave relacionada con la desnutrición y/o el abuso de laxantes. Después de evacuar por completo el colon por medio de un laxante, pueden pasar varios días antes de que ocurra una nueva evacuación normal. Entretanto, el paciente queda convencido de que está experimentando estreñimiento y puede continuar tomando el laxante.

Entre los trastornos hormonales ${ }^{11}$ se destaca la amenorrea, que generalmente coincide con el comienzo y es uno de los síntomas que más tardíamente desaparece. Puede ser primaria (paciente que todavía no había tenido su menarca) o secundaria y está vinculada con la desnutrición, el ejercicio y a sus efectos sobre el eje hipotálamo-hipofisiario-gonadal, pero también con aspectos psicológicos ya que en el $30 \%$ de los casos, la amenorrea precede el adelgazamiento y persiste cuando el peso se ha equilibrado. Los trastornos hormonales pueden conducir a una disminución de la densidad mineral ósea. En los hombres el bajo peso también se asocia con hipogonadismo, pudiendo disminuir los niveles séricos de testosterona.

La sexualidad puede ser objeto de una represión masiva, con ausencia de carga afectiva erógena del cuerpo. Si existe actividad sexual, parece realizarse sin placer, mecánicamente y como una manifestación de las conductas de control. No hay fatiga y sí hiperactividad motora asociada a una disminución de la duración del sueño y conductas ascéticas (caminar hasta el agotamiento, dormir en el piso, etc.). Hay mentiras, manipulación y cleptomanía en forma variable, sobre todo vinculadas con la comida (por ejemplo, robo de alimentos). La presencia de síntomas psicóticos deben hacer reconsiderar al diagnóstico.

\section{Bulimia nerviosa}

El ciclo de la bulimia es el atracón (cuadro 1) seguido de una $\mathrm{CCl}$. Diversos estados de ánimo disfóricos preceden y desencadenan las crisis; ya que la ingesta en sí reduce la sensación desagradable, pero luego provoca malestar físico y temor a aumentar de peso.

La forma clínica más habitual es la compulsiva normoponderal que evoluciona por crisis con vómitos. En contraste a la anorexia nerviosa, en que los pacientes tienen menos que el $85 \%$ de su peso normal, la mayoría de los pacientes bulímicos no altera su peso o lo preserva en valores ligeramente inferiores a los recomendados, pudiéndose encontrar conductas bulímicas en pacientes con sobrepeso e incluso obesos. Si bien hay preocupación exagerada y obsesionante por la imagen corporal, no habría una distorsión masiva de la percepción de la realidad del cuerpo.

La frecuencia de las crisis de atracones es variable, pudiendo llegar en casos muy graves a 15 diarias. La práctica puede darse en forma regular o producirse por períodos de semanas o meses, con intervalos de duración variable.

Las crisis van seguidas de $\mathrm{CCl}$, generalmente vómitos provocados, que con el tiempo, se vuelven casi automáticos, desapareciendo la necesidad de la estimulación mecánica. Evolucionado el trastorno, el paciente puede experimentar vivencias de despersonalización; estado que se acompaña de dolores físicos violentos, mayormente abdominales. Hay sentimiento de malestar, vergüenza, repugnancia de sí mismo, remordimientos y autoreproches. A pesar de este sufrimiento y de la conciencia de la anormalidad de la conducta, el malestar es rápidamente anulado.

Así como en la anorexia las complicaciones médicas aparecen como resultado de la restricción alimentaria y del descenso de peso, en la bulimia sobrevienen por el modo y la frecuencia de los atracones. De todas formas, el abuso de medicamentos, laxantes, diuréticos y anorexígenos pueden determinar complicaciones graves. Se describen las siguientes: 
Erosión del esmalte dentario, caries y úlceras o lesiones a nivel faríngeo producto de la exposición repetida al ácido gástrico contenido en los vómitos. Los vómitos frecuentes pueden determinar reflujo gastroesofágico,o síndrome de MalloryWeiss. Es frecuente la dispepsia y la constipación consecutiva al abuso de laxantes.

Las purgas recurrentes pueden determinar complicaciones electrolíticas: alcalosis metabólica en los casos graves y producto de los vómitos reiterados o por el uso de diuréticos; hipokalemia en un $5 \%$ de los pacientes bulímicos, con las posibles repercusiones cardiológicas. El hallazgo de hipokalemia en una paciente joven durante su control de salud es altamente específico de bulimia nerviosa, no estando recomendado realizar rastreo de esta condición en personas asintomáticas. Las pacientes con bulimia rara vez tienen complicaciones endócrinas, pudiendo observarse alteraciones menstruales que afectan la fertilidad. La mayoría mejora su sintomatología cuando se embaraza, recayendo una vez concluida la gestación.

\section{Sistemática de estudio}

Cuando se sospecha un trastorno alimentario, la evaluación apunta a tres objetivos principales: determinar si se trata de un verdadero trastorno de la alimentación o una variación de lo considerado "normal"; estimar la gravedad del cuadro y ubicar al paciente en alguno de los síndromes descriptos.

Es conveniente reservar un espacio de la entrevista para estar a solas con el paciente, sin embargo, hay puntos que deben ser interrogados a los pacientes y a sus familias. tiempo de evolución del problema (cuándo comenzaron las fluctuaciones del peso y conductas) pesos máximos y mínimos alcanzados, presencia de atracones y $\mathrm{CCl}$, historia menstrual; uso de fármacos tradicionales o de "preparados" que puedan contener anfetaminas, diuréticos, hormona tiroidea y/o benzodiazepinas. No hay que desaprovechar la posibilidad de citar a las familias cuando se sospecha el problema. Muchas veces hay preocupación familiar por ciertas conductas del paciente que son más evidentes en el hogar que en el consultorio médico, por lo que confían o delegan su detección en el equipo de salud. Sin embargo, lo más frecuente es que en el consultorio los pacientes nieguen o no manifiesten el problema.

\section{Cuestionarios específicos}

Sabiendo que un diagnóstico y un abordaje temprano mejoran el pronóstico de esta entidad, fueron desarrollados algunos cuestionarios para rastreo de trastornos alimentarios ${ }^{3}$. Sin embargo, hay dudas en cuánto a su aplicabilidad y confiabilidad. En primera instancia fue desarrollado el cuestionario EAT (Eating Attitude Test) y posteriormente el SCOFF ${ }^{13}$ (no validado en español) y que consta de cinco preguntas, cada respuesta afirmativa vale un punto; sugiriendo un resultado de dos puntos o más un probable caso de anorexia o bulimia nerviosa. Ver cuadro 3. Los creadores de este cuestionario reportan una sensibilidad de $100 \%$ para anorexia (IC95\%: 94,7-100\%) y/o bulimia (IC95\%: 92,6- 100\%); y un 87,5\% (IC95\% 79,2-93,4) de especificidad utilizando como "test de referencia a la entrevista estructurada con un psiquiatra entrenado y que utilice los criterios diagnósticos del DSMIVTR12-16.

Cuadro 3: traducción no validada al español, del test de actitud alimentaria (en inglés: Eating Attitude Test o EAT) para rastreo de anorexia y/o bulimia.
1- Luego de comer, ¿lo hace sentir enfermo tener la sensación de estar incómodamente lleno?

2- ¿Le preocupa haber perdido el control de cuánto está comiendo?

3- ¿Ha perdido en los últimos tres meses más de $6,3 \mathrm{~kg}$ ?

4- ¿Se siente gordo o gorda cuándo los demás le dicen que está muy delgado?

5- ¿Diría usted que la comida domina su vida?

Tanto el examen físico como los estudios complementarios, resultan de utilidad para evaluar el riesgo y descartar otras entidades causantes de la pérdida de peso. Sin embrago no sirven para hacer el diagnóstico, ya que a pesar de una importante disminución del peso pueden no hallarse alteraciones en los resultados de laboratorio.

Indagar los valores y las actitudes frente al propio peso, la imagen corporal y los hábitos alimentarios.

Recordar que puede hallarse anemia (mayormente normocítica normocrómica) leucopenia y más raramente trombocitopenia que se resuelven con la ganancia de peso. La deshidratación puede reflejarse en un aumento de urea sérica. Es frecuente la hipercolesterolemia y, en pacientes bulímicos, puede hallarse un aumento de la amilasa, probablemente como reflejo de la elevación de la isoenzima salival.

Tener especial atención al estado nutricional a través de la valoración del peso y de la talla, tomar los signos vitales, evaluar la función cardiovascular (electrocardiograma) el medio interno (sodio y potasio) y la función renal con el fin de detectar situaciones de peligro, por ejemplo alteraciones electrocardiográficas consecutivas a hipopotasemia. Si se sospecha de abuso de laxantes, evaluar el calcio sérico, el magnesio y el estado ácido base. Según las manifestaciones clínicas, el grado de sospecha y la gravedad, solicitar hemograma, proteinograma, función tiroidea, densitometría, urea, glucemia, colesterol y amilasa.

Son criterios de gravedad que pueden implicar la necesidad de una internación ${ }^{3}$ una disminución del peso mayor al 15\%; dificultad para cumplir indicaciones en el tratamiento ambulatorio; descompensación hidroelectrolítica (potasio plasmático menor a $3 \mathrm{mmol} / \mathrm{L}$ ) o psíquica (p. ej. intento o ideación suicida o psicosis) rápida y grave pérdida de peso refractaria al manejo ambulatorio; hipotensión o síncopes; pulso menor a 40 latidos por minuto; ortostatismo; arritmias o prolongación del intervalo QT.

\section{Diagnósticos diferenciales}

\section{Pérdidas de peso por otras causas}

Sospecharlas especialmente en mayores de $40 \mathrm{años}^{12}$. Se denomina "pérdida clínicamente importante" a más del $5 \%$ el peso durante seis meses. Puede ser útil preguntar: ¿la pérdida de peso es voluntaria o involuntaria? ¿hay aumento del apetito? (son pocas las causas de pérdida de peso y apetito aumentado) ¿desde cuándo comenzó la pérdida de peso?

Las causas más frecuentes de pérdida de peso involuntaria con aumento del apetito son el hipertiroidismo, la diabetes, los síndromes de malabsorción (recordar que pueden cursar sin diarrea) el feocromocitoma y el marcado aumento de la actividad física. En ausencia de aumento del apetito, pensar en cáncer, infección por el virus de inmunodeficiencia humana otras endocrinopatías, etc.

Trastornos psiquiátricos

En el trastorno depresivo mayor una pérdida de más del $5 \%$ del peso es un criterio diagnóstico. Sin embargo, los pacientes deprimidos no suelen tener deseos de una pérdida importante 
de peso o temor a ganarlo. Y, si bien es cierto que la depresión y la anorexia nerviosa comparten algunas características clínicas tales como sentimientos negativos, crisis de llanto, trastornos del sueño, rumiaciones obsesivas y tendencias suici-das ocasionales; existen varios rasgos distintivos.

En general, el paciente deprimido tiene poco apetito, mientras que el anoréxico afirma que tiene hambre (el apetito sólo declina en estadíos más graves). En contraste con la agitación depresiva, la hiperactividad de la anorexia es planeada y ritual. La preocupación por el valor calórico de los alimentos y recetas es típica de la anorexia. Además, en el deprimido no se advierte temor a la obesidad ni distorsión de la imagen corporal. En la depresión la autoestima se reduce, mientras que en la anorexia depende de la imagen del cuerpo y de los registros ponderales.

En la esquizofrenia puede observarse un comportamiento alimentario particular que determine una disminución del peso, pero rara vez se acompaña de temor a ganarlo o a alteraciones de la imagen corporal ${ }^{18}$.

En las fobias la evitación se limita a un determinado alimento o situación vinculable. En el trastorno obsesivo compulsivo, las obsesiones y las compulsiones pueden vincularse con la comida, siendo ésta una comorbilida frecuente. Pueden exhibir obsesiones y compulsiones no vinculadas a la comida, por ejemplo un excesivo temor a la contaminación.

En el trastorno dismórfico corporal hay preocupación por un defecto corporal imaginario o una distorsión exagerada de un defecto mínimo. Es decir que se establecerá una comorbilidad cuando la distorsión no esté relacionada con la forma o el tamaño del cuerpo (p. ej. preocupación excesiva por el tamaño de la nariz).

\section{Conductas alimentarias "grises"}

Muchas conductas alimentarias podrían ubicarse entre una conducta alimentaria "normal" y las formas claramente patológicas. La hiporexia puede ser constitucional o representar formas menores de anorexia mental, como respuesta a las múltiples demandas de la sociedad que valoriza la delgadez y las conductas de restricción alimentaria. El picoteo o "nibbling" por hedonismo alimentario, suele ocurrir como respuesta a los estímulos sociales de una alimentación abundante (galletitas, chocolate, snaks, etc.) pudiendo igualmente estar asociado a conductas alimentarias aberrantes. Otras conductas son las compulsiones alimentarias o "craving", que provocan placer y suelen involucrar al chocolate; el síndrome alimentario nocturno o "night eating syndrome" y los vómitos autoinducidos, que pueden presentarse aisladamente, constituyendo en si mismos la conducta valorizada ${ }^{19}$.

\section{Comorbilidades}

En la gran mayoría de los casos se encuentran trastornos comórbidos del eje I y del eje II del DSMIVTR ${ }^{3}$. De igual forma, algunos cuadros clínicos se asocian de manera temporal con trastornos de la conducta alimentaria y difieren su abordaje según esta relación ${ }^{14}$.

Existe mayor frecuencia de síntomas depresivos y de trastornos del estado de ánimo, especialmente distimia, en pacientes con diagnóstico de bulimia nerviosa. En la mayoría, el comienzo del trastorno del estado de ánimo comienza simultáneamente o a continuación de la bulimia. Como se puede observar en el cuadro 4 , la asociación con los trastornos del estado de ánimo es alta, sin embargo hay estados de inanición (con o sin anorexia nerviosa) que determinan síntomas como humor depresivo, irritabilidad, insomnio y disminución del interés sexual. Por lo tanto, se sugiere la reevaluación del cuadro junto con la recuperación nutricional.

Los rasgos obsesivo-compulsivos son frecuentes (relacionados y no relacionados a la comida). Muchos pacientes tienen pensamientos recurrentes vinculados con la comida; juntan recetas o acumulan alimento. La inanición puede producir este tipo de síntomas, pero cuando los pacientes exhiben obsesiones y compulsiones no relacionadas con comida, el peso, la silueta corporal; el diagnóstico de comorbilidad obsesivo compulsivo debería realizarse.

Existe invariablemente una asociación con trastornos de la personalidad. La anorexia parece más vinculada al grupo de trastornos de personalidad del grupo $\mathrm{C}$, mientras que la bulimia se relaciona más con el grupo $\mathrm{B}$. Dentro de este último grupo se encuentra el trastorno límite de la personalidad y son varias las características que suelen compartir pacientes bulímicos con pacientes con trastorno límite: inestabilidad afectiva, impulsividad, consumo de sustancias, conductas patológicas como el robo y tentativas de suicidio'. El pronóstico del trastorno alimentario, se ve muy influenciado por el trastorno comorbido.

Aquellos pacientes subtipo "purgativos" se asocian con mayor frecuencia a otros problemas en el ámbito del control de los impulsos, abuso de alcohol u otras drogas, labilidad emocional, intentos suicidas $\mathrm{y}$, a reunir criterios DSMIVTR para trastorno limite de la personalidad.

Cuadro 4: frecuencia de comorbilidades en el eje I del DSMIVTR

\begin{tabular}{|c|c|c|c|}
\hline \multirow[b]{2}{*}{ Diagnóstico } & \multicolumn{2}{|c|}{$\begin{array}{c}\text { Anorexia nerviosa } \\
\text { según subtipo }\end{array}$} & \multirow[b]{2}{*}{ Bulimia nerviosa } \\
\hline & Restrictivo & Purgativo & \\
\hline Algún trastorno afectivo & $57 \%$ & $100 \%$ & $100 \%$ \\
\hline Trastorno depresivo mayor & & $66 \%$ & \\
\hline Manía/hi pomana & 0 & $33 \%$ & $25 \%$ \\
\hline Algún trastorno de ansiedad & \multicolumn{3}{|c|}{50 a $65 \%$} \\
\hline Fobias & $43 \%$ & $11 \%$ & 0 \\
\hline Trastorno de pánico & \multicolumn{2}{|c|}{20 a $30 \%$} & \\
\hline Trastorno de ansiedad generalizada & \multicolumn{2}{|c|}{11 a $14 \%$} & $50 \%$ \\
\hline Trastorno obsesivo-compulsivo & \multicolumn{2}{|l|}{$14 \%$} & 50 a $56 \%$ \\
\hline Abuso/dependencia & \multicolumn{2}{|l|}{$14 \%$} & 33 a $50 \%$ \\
\hline Alguna comorbilidad & \multicolumn{3}{|c|}{70 a $100 \%$} \\
\hline
\end{tabular}

\section{Pronóstico}

El curso de los trastornos alimentarios es extremadamente variado en duración y gravedad, dependiendo de comorbilidades y el tiempo de evolución. El riesgo de muerte es más bajo para la bulimia que para los pacientes con diagnóstico de anorexia nerviosa Por el contrario, el buen soporte familiar y la detección temprana se asocian a un mejor pronóstico.

\section{Anorexia}

Algunos trabajos clasifican la evolución en buena, intermedia y mala. Si bien los datos son variables podrían resumirse en un $30 \%$ para cada una de ellas ${ }^{15}$. Otros autores presentan estadísticas más alentadoras en las que la recuperación llegaría hasta el $90 \%$. Probablemente estas diferencias sean consecutivas a la proveniencia de los pacientes sesgos de selección de los pacientes.

La anorexia nerviosa está marcada por períodos de recaídas, 
remisiones y evolución a bulimia nerviosa . Si bien el porcentaje de pacientes que podrían evolucionar a bulimia es muy variable (8 a $62 \%$ ) se señala que la gran mayoría que evoluciona, lo hace durante los primeros cinco años del diagnóstico de anorexia. Serían predictores de pasaje de anorexia a bulimia un alto grado de exigencia de los padres a los hijos; y de bulimia a anorexia, el abuso y la dependencia al alcohol y un bajo nivel de iniciativa personal ${ }^{16}$.

La detección y el abordaje tempranos mejoran el pronóstico e inversamente, cuanto más bajo es el peso o el índice de masa corporal al comienzo del tratamiento, mayor es el riesgo de un pronóstico desfavorable, sobre todo cuando el IMC es menor a $13^{10}$. Los mejores signos pronósticos están dados por un peso normal al alta del tratamiento agudo, el seguimiento por equipos con experiencia y la baja comorbilidad. El pronóstico no es peor en varones y la presencia de actividad sexual sería un factor de buen pronóstico en hombres.

La presencia de comorbilidades como los trastornos de la personalidad o depresión implican un peor pronóstico.

La mortalidad oscila entre $5,9 \%^{7}$ a $20 \%$ a los 20 años siendo las principales causas la inanición, suicidio y muerte súbita de causa cardiaca ${ }^{17}$.

\section{Bulimia}

La bulimia es un trastorno más variable en su gravedad, comorbilidad y tratamiento que la anorexia1. Un $70 \%$ de los casos tendría evolución favorable o intermedia. Si bien hay un $20 \%$ de pacientes con síntomas crónicos de bulimia (20\%) suelen ser individuos no tratados.

La existencia de comorbilidades como consumo de alcohol, trastorno límite de la personalidad, antecedentes de tentativas de suicidio o una evolución prolongada previamente al inicio del tratamiento son factores desfavorables.

Si los trastornos del humor, ansiedad u obsesivo-compulsivo preceden al TA generalmente se instalan por tiempo prolongado y requieren un abordaje específico; aunque igualmente los síntomas obsesivos y los depresivos suelen mejorar con la mejoría del TA. La tasa de mortalidad es de $0,3 \%$ a $0,5 \%$.

\section{Referencias}

1. Sadock BJ, Sadock VA, Kaplan HI: Kaplan \& Sadock's comprehensive textbook of psychiatry / editors, Benjamin J. Sadock, Virginia A. Sadock. Philadelphia, Lippincott Williams \& Wilkins, 2004.

2. Pearce JM: Richard Morton: origins of anorexia nervosa. Eur Neurol 2004; 52(4):191-2.

3. Diagnostic and Statistical Manual of Mental Disorders. Fourth Edition Text Revision. Washington DC. American Psychiatric Association, 2000.

4. Johnson JG, Cohen P, Kasen S, Brook JS: Childhood adversities associated with risk for eating disorders or weight problems during adolescence or early adulthood. Am J Psychiatry 2002; 159(3):394-400.

5. Corcos M AG, Bochereau D, Chambry et Jeammet PH.: Troubles des conduites alimentaires á ládolescence., in Encyclopédie Médico-Chirurgicale, vol 37-215-B-65. Paris, Elsevier, 2002, p 16p.

6. Rutsztein G: La Anorexia Nerviosa y el proceso de separación-individuación. Vertex. Rev. Arg. de Psiaquiat. 1998; IX(32):118-124.

7. Muris $\mathrm{P}$, Meesters $\mathrm{C}$, van de Blom W, Mayer B: Biological, psychological, and sociocultural correlates of body change strategies and eating problems in adolescent boys and girls. Eat Behav 2005; 6(1):11-22

8. Becker AE, Grinspoon SK, Klibanski A, Herzog DB: Eating disorders. N Engl J Med 1999; 340(14):1092-8.

9. Striegel-Moore RH, Dohm FA, Kraemer HC, Taylor CB, Daniels S, Crawford PB, Schreiber GB: Eating disorders in white and black women. Am J Psychiatry 2003; 160(7):1326-31

10. Mehler PS: Diagnosis and care of patients with anorexia nervosa in primary care settings. Ann Intern Med 2001; 134(11):1048-59.

11. Gidwani GP, Rome ES: Eating disorders. Clin Obstet Gynecol 1997; 40(3):601-15.

12. Mehler PS: Clinical practice. Bulimia nervosa. N Engl J Med 2003; 349(9):875-81.

13. Morgan JF, Reid F, Lacey JH: The SCOFF questionnaire: assessment of a new screening tool for eating disorders. Bmj 1999; $319(7223): 1467-8$

14. Palmer RL: Death in anorexia nervosa. Lancet 2003; 361(9368):1490.

15. Johnson CL, Lund BC, Yates WR: Recovery rates for anorexia nervosa. Am J Psychiatry 2003; 160(4):798; author reply 798.

16. Bulik CM, Sullivan PF, Fear J, Pickering A: Predictors of the development of bulimia nervosa in women with anorexia nervosa. J Nerv Ment Dis 1997; 185(11):704-7.

17. Sullivan PF. Mortality in anorexia nervosa. Am J Psychiatry 1995;152:1073-1074. 